Note

\title{
Teaching clinical reasoning and decision-making skills by visualizing the thought-process: an interdisciplinary gaming project involving honours students from veterinary and computing sciences
}

\author{
Andrea A. G. Laumen ${ }^{1 *}$, Milou van Velzen ${ }^{1}$, Ivan Veul ${ }^{2}$, Ineke Lam³ ${ }^{3}$, Wolfgang O. Hürst ${ }^{4}$,
} Yvonne R. A. van Zeeland ${ }^{5}$

1. Faculty of Veterinary Medicine, Utrecht University, Utrecht, The Netherlands

2. Faculty of Sciences, Utrecht University, Utrecht, The Netherlands

3. Centre for Teaching and Learning, Utrecht University, Utrecht, The Netherlands

4. Department of Information and Computing Sciences, Faculty of Sciences, Utrecht University, Utrecht, The Netherlands

5. Department of Clinical Sciences of Companion Animals, Faculty of Veterinary Medicine, Utrecht University, Utrecht, The Netherlands

*Correspondence: a.a.g.laumen@uu.nl

Received: 11 September 2017; Accepted: 23 October 2017; Published: 7 November 2017

Keywords: clinical reasoning; serious game; interdisciplinary project

\section{Introduction}

Clinical reasoning (figure 1 ) is the capability to link one's own observations and interpretations to medical knowledge, thereby rendering this an essential skill to master in professional veterinary and medical health sciences (Delany \& Golding, 2014). Teaching and learning this skill can be challenging for both teacher and student, because of the complexity of the thought process (Delany \& Golding, 2014). Moreover, the limited availability of real patients necessitates teachers to rely on less-realistic paper casuistries for practicing purposes. However, serious games, which are designed for the simulation of real-life situations, may offer a solution (Johnsen et al., 2016).

In health sciences, serious games can be used to provide students with the opportunity to practice their clinical reasoning and decision-making skills in an accessible, safe and authentic environment (Delany \& Golding, 2014; Johnsen et al., 2016). Several of these games have already been developed and used in the medical sciences (Delany \& Golding, 2014; Johnsen et al., 2016; Koivisto et al., 2016; Graafland, Schraagen \& Schijven, 2012), but in veterinary medicine, their availability is scarce. As a result, an interdisciplinary serious gaming project was set up to develop a program with a group of veterinary and computing science honours students. 


\section{Clinical reasoning}

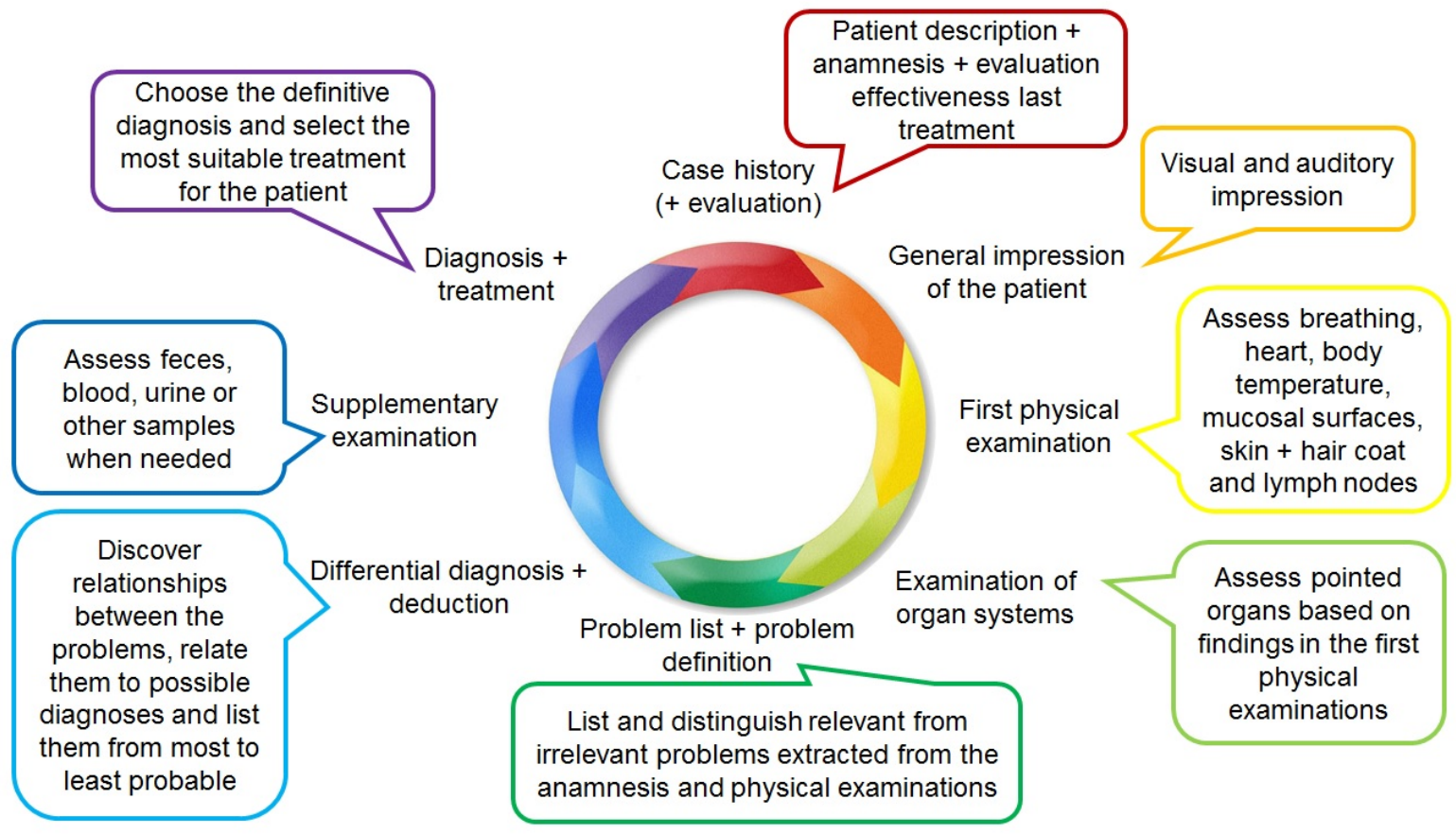

Source: Based on Mather (2013) and the Course Information 'Lijn 3', Faculty of Veterinary Medicine, Utrecht University, the Netherlands

The project aimed to develop a serious game for veterinary students to practice their clinical reasoning skills using interactive casuistries, while at the same time offering the participating students an opportunity to broaden their horizon and gain insight in the work processes of a different discipline, deepen the veterinary students' knowledge regarding the structure of the clinical reasoning process and familiarize the computing science students with the process of designing software for a commissioning party. During the project, each group was assigned its own tasks, whereby the veterinary students were primarily responsible for writing a case script and testing of the functionality of the game, while the computing science students (aided by students from the art faculty) were responsible for software development and lay-out of the program.

This project was done in an honours setting since the development of the game and its software required the input of students to correspond with the knowledge, expertise and insights of the students. Thinking about the design and contents of the game challenged the honours student, corresponding with our vision on honours programs in veterinary medicine, in which we want to stimulate creativity, ingenuity and interdisciplinarity. Furthermore, the project added extra content to the thinking process and patterns in the education of clinical reasoning.

\section{Methods}

Development of the program took place using the MoSCoW method (Miranda, 2011), starting with the determination of the minimal requirements for a working and accessible 
game (so-called 'Must haves'). In addition, 'Should haves' (i.e. desirable, but not necessary to obtain a functional product), 'Could haves' (i.e. requirements that are only included if time permits) and 'Won't haves' (i.e. requirements that will not be incorporated in the current project, but can be of interest for further development) were drawn up. During weekly to biweekly meetings, the veterinary and computing science students got together with teachers from both disciplines and educational advisors to discuss the design and development of the program and provide feedback on each other's activities. Computing science students furthermore visited the veterinary medicine faculty and participated in lessons to help illustrate the process.

\section{Results}

Six months after the start of the project, the first prototype of the serious game software was completed. This software, called 'Furo', allows teachers to program new clinical reasoning cases that allow the students to systematically go through the different steps of the clinical thought process, while receiving feedback following each completed step. Although further testing and development of the software is needed, this initial prototype provides an excellent example of the possibilities that serious gaming may provide to educators and students to facilitate teaching of clinical reasoning, as well as the advantages that the participation in an interdisciplinary project may have for the professional development of the (honours) student.

\section{Discussion}

One of the major challenges during this project was to attune the technical languages and working methods used in the different disciplines. This was greatly facilitated by having the students from computational science take part in the lectures at the veterinary faculty, helping them to visualize the thought process of clinical reasoning. Their technical breakdown of the process in turn helped veterinary students in their understanding of clinical reasoning. Moreover, the veterinary students' understanding of the clinical reasoning process was greatly enhanced by having the students design the case plot, including the questions, correct answers and distractors.

Other challenges faced during the project included problems of a logistical nature such as planning of the meetings, as many students had different class schedules. Given the size of each of the groups, it was quickly decided to hold weekly meetings at one of the two faculties, whereby only a small (variable) delegation of students from the other faculty was present to sit in on the meetings of the other group and give updates to the other groups. For this purpose it was essential to appoint group leaders for each group. Appointing group leaders was furthermore found beneficial for planning and communication, which were both found to be key factors to enable streamlining of the process, as both groups were highly dependent on each other's results. When one group didn't meet their milestones, the other group also couldn't continue. Group leaders furthermore ensured division of the work among smaller working groups, with each group member having their own responsibilities and being aware of that of others, which enabled good progress to be made.

It was valuable to work in an honours setting during this project. The added value of the setting was characterized by inter alia creative solutions, e.g. by the returning of the frames with the pet-owner in the slot-phase to round up the game, in order to be able to visualize 
all phases of the clinical reasoning process within the limited available time. Other advantages of the honours setting, in comparison with a setting of students from the regular curriculum, were the quick understanding of the information provided by the other discipline. Besides, since honours students are critical thinkers, this setting created a continuous critical evaluation of the encountered problems, which in turn led to quick solving of the problems. Another important educational aspect for the honours students was the interdisciplinary collaboration; they had to learn how to make certain issues understandable for the other discipline. Furthermore, organisation of the meetings and working and discussing in an efficacious way contributed to the professional attitude of the honours student.

\section{Conclusions}

The participating veterinary students indicated that they got more insight in the clinical reasoning process and both veterinary and computing science students mentioned that they got a better understanding of the work of the other discipline. The students from the regular curriculum testing the program stated they better remembered the subject matter. The game is currently in a test phase, but is further developed from 2016 onwards. New cases have been created already and the possibilities to make English versions are currently under discussion.

\section{References}

Delany, C., \& Golding, C. (2014). Teaching clinical reasoning by making thinking visible: an action research project with allied health clinical educators. BMC medical education, 14(1), 1. doi: 10.1186/1472-6920-14-20

Graafland, M., Schraagen, J. M., \& Schijven, M. P. (2012). Systematic review of serious games for medical education and surgical skills training. British journal of surgery, 99(10), 13221330.

Johnsen, H. M., Fossum, M., Vivekananda-Schmidt, P., Fruhling, A., \& Sletteb $\varnothing$, Å. (2016). Teaching clinical reasoning and decision-making skills to nursing students: Design, development, and usability evaluation of a serious game. International journal of medical informatics, 94, 39-48. http://dx.doi.org/10.1016/i.ijmedinf.2016.06.014

Koivisto, J. M., Multisilta, J., Niemi, H., Katajisto, J., \& Eriksson, E. (2016). Learning by playing: A cross-sectional descriptive study of nursing students' experiences of learning clinical reasoning. Nurse Education Today, 45, 22-28.

Mather, C. (2013). Clinical Reasoning resources for professional experience facilitators [blog]. Available online: https://blogs.utas.edu.au/snm-pep/2013/03/26/clinical-reasoningresources-for-professional-experience-facilitators/ (accessed 1 October 2017)

Miranda, E. (2011). Time boxing planning: Buffered Moscow rules. ACM SIGSOFT Software Engineering Notes, 36(6), 1-5. 